Case
Reports

Dheeraj Khurana, MD

Jaffar Raza, MD

Sunil Abrol, MD

Neil L. Coplan, MD
Key words: Cardiac tamponade/etiology; device removal; equipment failure foreign-body migration/ complications/diagnosis/etiology/surgery; heart injuries/ etiology; myocardial infarction/etiology; risk factors; treatment outcome; vena cava filters/adverse effects; vena cava, inferior/injuries

From: Department of Cardiology (Drs. Coplan, Khurana, and Raza), Lenox Hill Hospital, New York, New York 10075; Department of Cardiology (Drs. Khurana and Raza), Jamaica Hospital and Medical Center, Jamaica, New York 11418; and Department of Cardiovascular Surgery (Dr. Abrol), Brookdale University Hospital and Medical Center, Brooklyn, New York 11212

Address for reprints: Dheeraj Khurana, MD Department of Cardiology, Lenox Hill Hospital, 100 E. 77th St., New York, NY 10075

E-mail: dheeraj2302@yahoo.com

(C) 2015 by the Texas Heart ${ }^{\circledR}$ Institute, Houston

\section{Fractured Inferior Vena Cava Filter Strut}

\author{
Presenting with ST-Segment \\ Elevation and Cardiac Tamponade
}

The fracture of an inferior vena cava filter strut and its migration to the heart is a rare sequela of implanted inferior vena cava filters. Perforation through the right ventricle into the pericardium with resultant cardiopulmonary compromise is even less frequent. We report the case of a 53-year-old man who presented with chest pain and hypotension consequent to cardiac tamponade. A fractured inferior vena cava filter strut had migrated and perforated his right ventricle. The fractured strut was successfully removed by means of cardiac surgery. Inferior vena cava filters should be placed when necessary to minimize the risk of pulmonary embolism, and regular radiologic monitoring should be performed; however, the eventual extraction of retrievable filters should be considered. In addition to discussing the patient's case, we briefly review the relevant medical literature. (Tex Heart Inst J 2015;42(2):181-3)

I nferior vena cava (IVC) filters were first introduced in 1967, to prevent pulmonary embolism (PE) when anticoagulation is contraindicated or has failed in patients who have venous thromboembolic disease. In addition, IVC filters are used prophylactically in patients who have a high risk of PE. Implanted IVC filters are associated with such long-term sequelae as thrombotic occlusion of the IVC, vena cava perforation, and filter dislocation and migration.

Inferior vena cava filter fracture has been reported in less than $1 \%$ of cases, ${ }^{2}$ and the fracture of an IVC filter strut with its subsequent migration to the heart is apparently even less frequent. ${ }^{3}$ We report the case of a man who presented with ST-segment elevation and cardiac tamponade after an IVC filter strut fractured, migrated, and perforated his right ventricle (RV). We also briefly review the relevant medical literature.

\section{Case Report}

In November 2010, a 53-year-old man presented at the emergency department 5 hours after the sudden onset of left-sided chest pain. His medical history included intracranial hemorrhage after head trauma 5 years earlier; afterwards, he had experienced seizures that were treated with antiepileptic medications. He had also had a Bard G2 ${ }^{\circledR}$ retrievable IVC filter placed during his hospitalization for the intracranial hemorrhage; however, he did not report this to the physicians at the current admission. His vital signs on admission included a blood pressure of $90 / 56 \mathrm{mmHg}$, a heart rate of 101 beats/min, a respiration rate of 19 breaths $/ \mathrm{min}$, and an oxygen saturation of $98 \%$ on room air. An electrocardiogram showed sinus tachycardia at the rate of 117 beats/min and ST-segment elevation in the inferior leads.

The patient was started on intravenous fluids and a dopamine infusion and was urgently taken to the cardiac catheterization laboratory. During angiography, the patient became hemodynamically unstable; his systolic blood pressure fell to $60 \mathrm{mmHg}$. Left-sided heart catheterization was performed through femoral access. The left main coronary artery was normal. There was an eccentric $70 \%$ stenosis in the proximal left anterior descending coronary artery (LAD) and a $60 \%$ stenosis in the mid-LAD. The first obtuse marginal branch was large and had an $80 \%$ stenosis. The ostial right coronary artery (RCA) had a 75\% stenosis, and the mid RCA had a 70\% stenosis. The patient's left ventricular ejection fraction (calculated by means of contrast ventriculography) was 0.70 . During fluoroscopy, a small metal object was visible in the 
RV territory, but it was not clear if this was inside or outside of the heart. Of note, right-sided heart catheterization showed an equalization of diastolic pressures in all chambers of the heart, suggesting cardiac tamponade. An urgent bedside echocardiogram revealed a small pericardial effusion and evidence of cardiac tamponade.

The patient was taken to the operating room and underwent pericardial fenestration, with drainage of 450 $\mathrm{mL}$ of blood. His blood pressure improved after the procedure. A computed tomogram (CT) of the chest and abdomen revealed a slightly curved foreign body. It was partially in the RV and had traversed the anterior RV wall into the pericardium (Figs. 1 and 2). An abdominal CT showed an IVC filter with a missing anterior strut (Fig. 3). The patient underwent surgical extraction of the IVC filter strut, which had perforated the diaphragmatic surface of the RV. He had an uneventful recovery and was discharged from the hospital after 5 days.

\section{Discussion}

This was a rare case of IVC filter fracture that led to cardiac tamponade. Inferior vena cava filters are used to prevent $\mathrm{PE}$ in patients in whom anticoagulation therapy is ineffective or contraindicated. The caliber of the IVC varies with respirations, cardiac function, and intravascular volume; these factors can contribute to metal fatigue and strut fracture, although IVC strut fracture is apparently very infrequent.

When implanted for long periods $(1,946 \mathrm{~d}$ in our patient), IVC filters theoretically sustain more metal stress. Lynch and colleagues ${ }^{4}$ retrospectively reviewed the removal of Bard G2 IVC filters in 174 patients whose implantation periods were longer or shorter than 180 days. Of $174 \mathrm{G} 2$ filters, 170 were removed (success rate, $97.7 \%)$. Of the 6 filters that had fractured (3.4\%), all had been in place longer than 180 days. ${ }^{4}$

Inferior vena cava filter migration to the right atrium and the RV has been described. In 1996, James and associates ${ }^{5}$ reviewed 22 cases of Greenfield IVC filter migration to the heart: 15 devices had embolized to the right atrium, one to the RV, 3 to the right pulmonary artery, one to the left pulmonary artery, and one to the "atrium." 5 In the 22nd patient, who died, the IVC filter was attached to the myocardial wall. Ten patients were asymptomatic, 4 presented with arrhythmias, and one had pericardial tamponade. In 2004, Izutani and co-authors ${ }^{6}$ reported 12 cases of IVC filter migration to the heart ( 5 to the right atrium, 5 to the $\mathrm{RV}$, and 2 to the pulmonary artery). Half of these patients were asymptomatic. No filter-related death was recorded, although 4 patients had arrhythmias.

Nicholson and colleagues ${ }^{7}$ conducted a retrospective study of 80 patients to determine the prevalence of fracture and embolization of the Bard Recovery ${ }^{\circledR}$ and G2

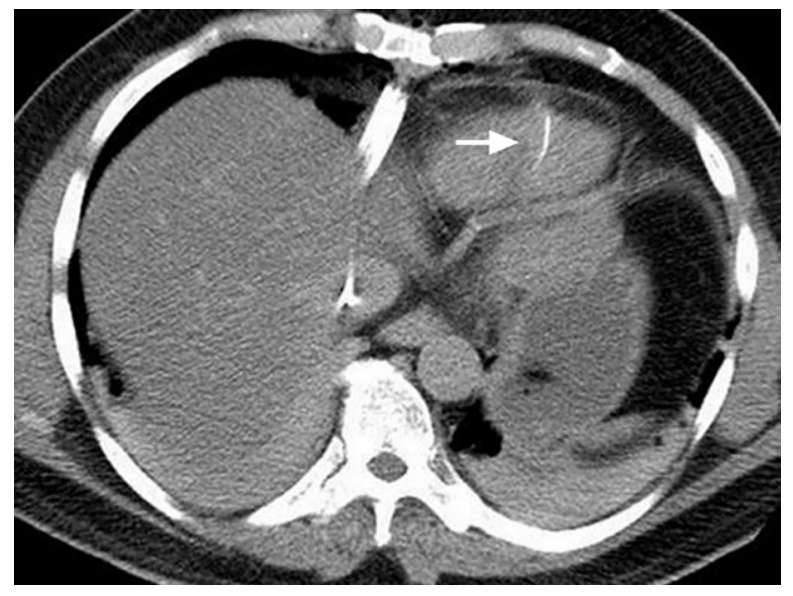

Fig. 1 Computed tomogram of the chest and abdomen shows a fractured inferior vena cava filter strut (arrow) in the right ventricle.

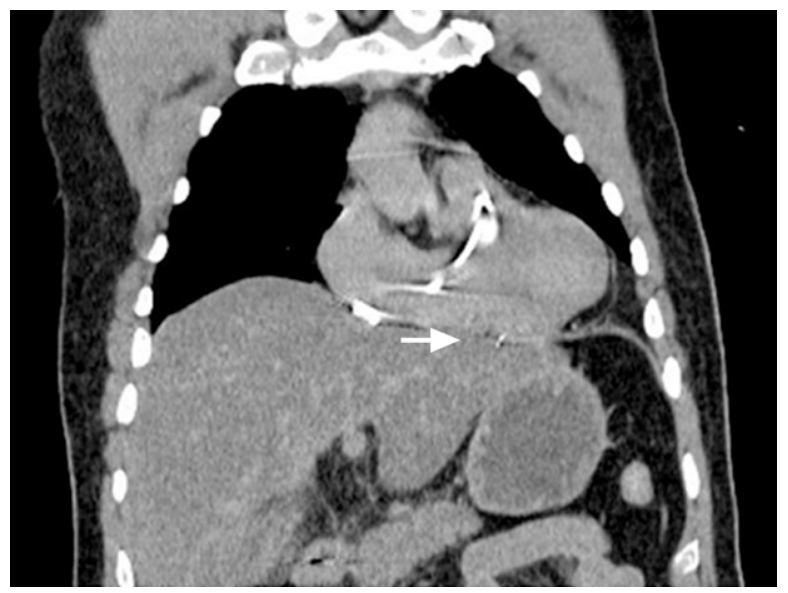

Fig. 2 Longitudinal section of a computed tomogram of the chest and abdomen shows a fractured inferior vena cava filter strut (arrow) at the inferior border of the heart.

vena cava filters. Thirteen of 80 patients had at least one strut fracture (16\%). Of the 28 Recovery filters, 7 had at least one fractured and embolized strut (25\%). Six of 52 G2 filters fractured (12\%). The average time between Recovery filter implantation and evaluation of filter integrity was 1,498 days; for the G2 filter, it was 717 days. The average placement durations for patients in whom fracture was observed in the Recovery and G2 groups were nearly identical to those of all patients in those respective groups. The authors suggested that filter fractures might be a class effect and not limited to a specific design. Nevertheless, the effects of such fractures can vary. For example, Bard retrievable filters have radially distributed arms that are connected only at a single point. Therefore, a single fracture would result in a free fragment capable of embolization and potentially dangerous consequences, as in our patient. 


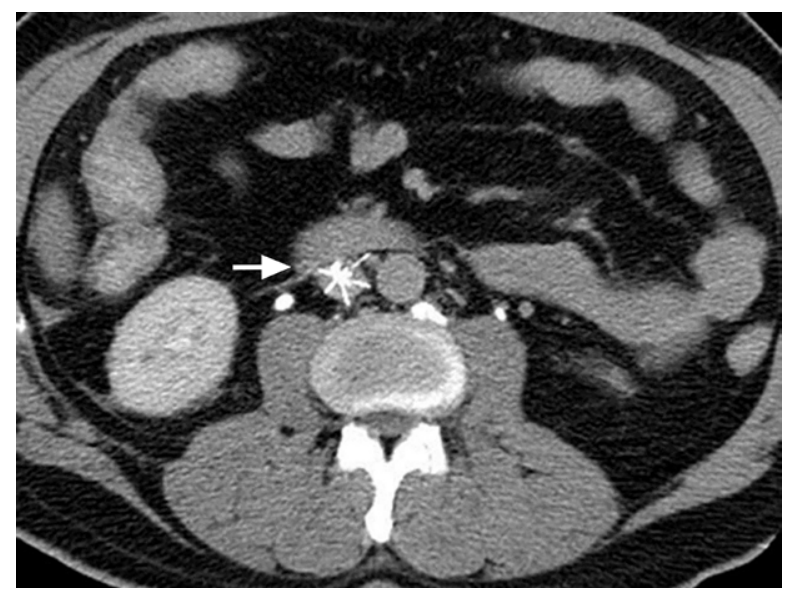

Fig. 3 Computed tomogram of the abdomen shows the missing filter strut anteriorly (arrow).

Because filter fragmentation and embolization might occur secondary to metal fatigue and be related to the duration of implantation, it is prudent that patients be evaluated regularly for evidence of filter fracture. In addition, each patient who has a retrievable filter should be considered for filter retrieval. Vijay and associates ${ }^{8}$ retrospectively reviewed a database of fractured IVC filters, to understand the risks of IVC filter fracture and embolization and the safety of removing fractured filters. In 63 fractured Recovery, G2, and G2 Express $^{\circledR}$ IVC filters identified in 548 patients, the overall fracture rate was nearly $12 \%$. The occurrence of fracture increased with longer filter implantation durations. The success rate for removal of the nonfractured main body was $98.4 \%$, and it was $53.4 \%$ for fractured arms or legs. The authors concluded that such removals can be safely and effectively achieved. ${ }^{8}$ The U.S. Food and Drug Administration has recommended that physicians who perform implantations and clinicians who monitor patients with retrievable IVC filters consider filter removal as soon as the risk of PE has diminished. Consideration of the risks and benefits of filter placement in each patient is encouraged.

The presentation of IVC filter-strut fracture can be varied and misleading. Our patient's chest pain and ST-segment elevation raised suspicion of myocardial infarction and prompted a diagnostic angiogram. Echocardiography followed by CT is ideal for the diagnosis of possible filter fracture and migration, and cardiac surgery with or without cardiopulmonary bypass is the treatment of choice for extracting fractured filter struts.

\section{References}

1. Imberti D, Ageno W, Dentali F, Donadini M, Manfredini R, Gallerani M. Retrievable vena cava filters: a clinical review. J Thromb Thrombolysis 2012;33(3):258-66.

2. Kinney TB. Update on inferior vena cava filters. J Vasc Interv Radiol 2003;14(4):425-40.
3. Ferris EJ, McCowan TC, Carver DK, McFarland DR. Percutaneous inferior vena caval filters: follow-up of seven designs in 320 patients. Radiology 1993;188(3):851-6.

4. Lynch FC, Kekulawela S. Removal of the G2 filter: differences between implantation times greater and less than 180 days. J Vasc Interv Radiol 2009;20(9):1200-9.

5. James KV, Sobolewski AP, Lohr JM, Welling RE. Tricuspid insufficiency after intracardiac migration of a Greenfield filter: case report and review of the literature. J Vasc Surg 1996; 24(3):494-8.

6. Izutani H, Lalude O, Gill IS, Biblo LA. Migration of an inferior vena cava filter to the right ventricle and literature review. Can J Cardiol 2004;20(2):233-5.

7. Nicholson W, Nicholson WJ, Tolerico P, Taylor B, Solomon $S$, Schryver T, et al. Prevalence of fracture and fragment embolization of Bard retrievable vena cava filters and clinical implications including cardiac perforation and tamponade. Arch Intern Med 2010;170(20):1827-31.

8. Vijay K, Hughes JA, Burdette AS, Scorza LB, Singh H, Waybill PN, Lynch FC. Fractured Bard Recovery, G2, and G2 express inferior vena cava filters: incidence, clinical consequences, and outcomes of removal attempts. J Vasc Interv Radiol 2012;23(2):188-94.

9. U.S. Food and Drug Administration [Internet]. Inferior vena cava (IVC) filters: initial communication: risk of adverse events with long term use. Available from: www.fda. gov/Safety/MedWatch/SafetyInformation/SafetyAlertsfor HumanMedicalProducts/ucm221707.htm [2010 Aug 9; cited 2013 Aug 1]. 